\section{To PRP or not?}

\section{Lars Engebretsen, 1,2,3 Kathrin Steffen ${ }^{1,2}$}

Was the Super Bowl XLIII really won by platelet-rich plasma (PRP)? In February 2009, Pittsburgh Steelers wide receiver Hines Ward, who suffered a medial knee ligament injury just a week before the big event, caught a pass which helped the Steelers win the Championship. Judging from the press clippings in the USA over the following week, it was PRP, which did it all! The injections jumpstarted the widespread use of PRP we now observe in the USA. Long before this, of course, PRGF (plasma rich in growth factors) had been introduced with much basic and some clinical research for maxillofacial surgery by Dr Eduardo Anitua in Vitoria, Spain. ${ }^{1}$ After first having published impressive results from his own research field, his group followed up with the first studies in sports medicine as well. ${ }^{2}$ In 2007, Peter A M Everts from Eindhoven in The Netherlands defended his impressive thesis on 'Autologous platelet-leucocyte enriched gel. Basics and efficacy,'3 where he used the system to support soft tissue and bone healing. We urge users of PRP to read the original works by Anitua and Everts filled with historic and recent science on PRP.

${ }^{1}$ IOC Medical Commission, Lausanne, Switzerland ${ }^{2} 0$ slo Sports Trauma Research Center, Department of Sports Medicine, Norwegian School of Sport Sciences, Oslo, Norway

${ }^{3}$ Oslo University Hospital, Faculty of Medicine, University of Oslo, Norway

Correspondence to Professor Lars Engebretsen, Oslo Sports Trauma Research Center, Department of Sports Medicine, Norwegian School of Sport Sciences, Norway; lars.engebretsen@medisin.uio.no
So why aren't we all happy? Finally, a method that will accelerate the healing of muscle, tendon, bone and cartilage injuries, shorten the rehabilitation time after musculoskeletal injuries and rejuvenate sleeping cells? Well, it seems that even though more is better, much more may not be so good, at least not in every term of the word. The issues of preparation procedure, with or without leucocytes, volume of autologous blood, the concentration of growth factors, method of delivery, dose and frequency, the effect of local anaesthesia and antiinflammatory medication are all unresolved issues. Even more importantly, the risks of side effects have not been fully elucidated, even though it all looks good at this stage.

This issue has a comprehensive review of PRP treatment in sports medicine. ${ }^{4}$ To discuss the use of PRP in a clinical setting, and the need for further research, the International Olympic Committee (IOC) assembled an expert group in May 2010 to critically review the current state of PRP treatment among athletes, aiming to provide recommendations for clinicians, athletes and individual sports governing bodies. The purpose of this consensus paper was furthermore to review the evidence for the clinical effectiveness of PRP, its ergogenic potential and safety, and attempt to reconcile any possible disparity between its increasing popularity and the underlying science supporting its use.

Our readers should be aware that the use of PRP in tissue healing and regeneration may open a new area in regenerative medicine, but also that there remains a large amount of work toward understanding the mechanism of action of $\mathrm{PRP}$ in the regeneration and repair process of a given tissue. Firm recommendations on the effectiveness of PRP in the clinical setting to support the healing processes of muscle, tendon, ligament and cartilage injuries cannot be given. Results of clinical studies on PRP are difficult to interpret, as the methodological quality of published investigations varied substantially. More attention should be paid to the use of solid methods when designing, performing and reporting clinical trials.

The final recommendation of the IOC consensus group is to proceed with caution in the use of PRP in clinical practice of sports medicine. The group believes that more work on the basic science needs to be undertaken and that greater rigour should be implemented in developing robust clinical trials to demonstrate the efficacy of PRP.

\section{Competing interests None.}

Provenance and peer review Commissioned; not externally peer reviewed.

Accepted 5 October 2010

Br J Sports Med 2010;44:1071.

doi:10.1136/bjsm.2010.080390

\section{REFERENCES}

1. Anitua E. Plasma rich in growth factors: preliminary results of use in the preparation of future sites for implants. Int J Oral Maxillofac Implants 1999:14:529-35.

2. Anitua E. A Biological Approach to Implantology. Vitoria, Spain: Team Work Media Espania, 2009.

3. Everts AMP. Autologous platelet-leukocyte enriched gel. Basics and efficacy. Thesis, University of Eindhoven, The Netherlands, 2007.

4. Engebretsen L, Steffen K, Alsousou J, et al. IOC consensus paper on the use of platelet-rich plasma (PRP) in sports medicine. Br J Sports Med 2010:44:1072-1081. 